\title{
ON PERMANENT VECTOR-LINES IN $N$ DIMENSIONS
}

\section{J. L. SYNGE}

Prim and Truesdell have recently given a simple vector proof of Zorawski's condition for the permanence of vector-lines in a moving fluid. ${ }^{1}$ Their argument is necessarily by its form confined to threedimensional space, and it seems of interest to investigate the problem in a Euclidean space of $N$ dimensions, using a slightly different approach.

We consider a Euclidean space $E_{N}$ with rectangular cartesians $x_{i}$. Two vector fields are given, $v_{i}(x, t), c_{i}(x, t)$, where $t$ is a parameter (the time); we shall call $v_{i}$ the primary and $c_{i}$ the secondary vector fields; $v_{i}$ plays the part of velocity.

Any vector field defines for given $t$ a congruence of curves in an obvious way, the direction of the curve at each point coinciding with that of the vector field. We are not particularly interested here in the congruence defined by the primary vector field (stream-lines), and we shall use the expression vector-lines to refer exclusively to the congruence defined by the secondary field, that is, those curves which satisfy

$$
c_{i} d x_{i}-c_{j} d x_{i}=0 .
$$

We use the primary field to generate an infinitesimal transformation $d x_{i}=v_{i}(x, t) d t$, the history of an individual particle being obtained by integrating these equations. Any curve at $t=t_{0}$ will thus generate as its history a succession of curves formed always of the same particles; this succession of curves may be expressed by

$$
x_{i}=f_{i}(\theta, t)
$$

where $\theta$ is a parameter which remains constant as we follow a particle. We have then the equations

$$
\partial f_{i} / \partial t=v_{i}, \quad \partial f_{i} / \partial \theta=\lambda_{i},
$$

where $\lambda_{i}$ is a vector tangent to the instantaneous position of the moving curve.

If we follow the history of a curve $C$ which moves with the fluid in the above sense and which is a vector-line at $t=t_{0}$, it will not in general remain a vector-line. To investigate the conditions under which

Received by the editors July 24, 1950.

${ }^{1}$ R. Prim and C. Truesdell, Proceedings of the American Mathematical Society vol. 1 (1950) pp. 32-34. 
it does so remain, we define a skew-symmetric tensor by

$$
\Omega_{i j}=c_{i} \lambda_{j}-c_{j} \lambda_{i} .
$$

Then $\Omega_{i j}=0$ is a necessary and sufficient condition that $C$ should be a vector-line.

Now $\Omega_{i j}$ is a function of $\theta$ and $t$, and if we write

$$
c_{i}(x, t)=g_{i}(\theta, t) \quad \text { when } \quad x_{i}=f_{i}(\theta, t),
$$

we have

$$
\Omega_{i j}(\theta, t)=g_{i} \partial f_{j} / \partial \theta-g_{j} \partial f_{i} / \partial \theta
$$

We proceed to examine how $\Omega_{i j}$ changes as we move with the fluid, its rate of change being $\partial \Omega_{i j} / \partial t$.

For arbitrary variations in the 2-space (2) we have

$$
\begin{aligned}
\delta c_{i} & =c_{i, k} \delta x_{k}+\left(\partial c_{i} / \partial t\right) \delta t \\
& =c_{i, k}\left(\lambda_{k} \delta \theta+v_{k} \delta t\right)+\left(\partial c_{i} / \partial t\right) \delta t \\
& =\left(\partial g_{i} / \partial \theta\right) \delta \theta+\left(\partial g_{i} / \partial t\right) \delta t,
\end{aligned}
$$

where the comma denotes partial differentiation and the summation convention is used. Hence

$$
\partial g_{i} / \partial \theta=c_{i, k} \lambda_{k}, \quad \partial g_{i} / \partial t=c_{i, k} v_{k}+\partial c_{i} / \partial t .
$$

Thus,

$$
\frac{\partial}{\partial t}\left(g_{i} \partial f_{j} / \partial \theta\right)=\left(c_{i, k} v_{k}+\partial c_{i} / \partial t\right) \lambda_{j}+c_{i} v_{j, k} \lambda_{k}
$$

since

$$
\frac{\partial}{\partial t}\left(\frac{\partial f_{j}}{\partial \theta}\right)=\frac{\partial}{\partial \theta} v_{j}=v_{j, k} \lambda_{k} .
$$

Accordingly we obtain from (6)

$$
\frac{\partial}{\partial t} \Omega_{i j}=\lambda_{q}\left(A_{q i j}-A_{q j i}\right)
$$

where

$$
A_{q i j}=\delta_{j q}\left(c_{i, k} v_{k}+\partial c_{i} / \partial t\right)+c_{i} v_{j, q} .
$$

If the curve in question is a vector-line which moves with the fluid, we have $\lambda_{q}=\phi c_{q}$, where $\phi$ is a scalar factor, and also $\partial \Omega_{i j} / \partial t=0$. Hence, by (11), 


$$
c_{q}\left(A_{q i i}-A_{q j i}\right)=0
$$

is a necessary condition for the permanence of vector-lines. It is also sufficient, as we see from consideration of the order of the equations involved and the fact that if (13) holds, then $\Omega_{i j}=0$ implies $\partial \Omega_{i j} / \partial t$ $=0$.

Hence we have this result: $A$ necessary and sufficient condition for the permanence of the vector-lines of $c_{i}$ is (13), or equivalently, that the tensor $c_{q} A_{q i j}$, that is,

$$
c_{j}\left(c_{i, k} v_{k}+\partial c_{i} / \partial t\right)+c_{i} v_{j, k} c_{k},
$$

shall be symmetric.

Dublin Institute for Advanced Studies

\section{NOTE ON A THEOREM IN SUMMABILITY}

J. D. HILL

Let $T$ denote a regular matrix method of summability in the complex domain, that is to say, a transformation of the form

$$
t_{n}=\sum_{k=1}^{\infty} a_{n k} s_{k} \quad(n=1,2,3, \cdots),
$$

having the property that the convergence of $\left\{s_{k}\right\}$ to $s$ always implies the existence of $t_{n}$ for each $n$ and the convergence of $\left\{t_{n}\right\}$ to $s$. It is well known that the following conditions of Silverman-Toeplitz are necessary and sufficient in order that $T$ be regular: $a_{n k}=o(1)(n \rightarrow \infty$; $k=1,2,3, \cdots) ; \sum_{k=1}^{\infty} a_{n k}=1+o(1)(n \rightarrow \infty) ;$ and

$$
\sum_{k=1}^{\infty}\left|a_{n k}\right|=O(1) \quad(n \rightarrow \infty) .
$$

The following theorem was established recently by Henstock [2].1

TheOREM (HeNstock). Let $y \equiv\left\{z_{k}\right\}$ be a given bounded sequence of complex numbers. Then there exist denumerably many sequences of

Received by the editors July $5,1950$.

${ }^{1}$ Numbers in brackets refer to the references at the end of the paper. 